\title{
AN ENERGY-REPLICATING CLASS OF NON-NORMALISABLE SUPERCOHERENT STATES
}

\author{
R.F. BISHOP, M.C. BOSCÁ ${ }^{1}$ and M.F. FLYNN \\ Department of Mathematics, Institute of Science and Technology, University of Manchester, \\ P.O. Box 88, Manchester M60 1QD, UK
}

Received 23 May 1988; revised manuscript 6 July 1988; accepted for publication 10 August 1988 Communicated by A.P. Fordy

\begin{abstract}
We investigate natural supercoherent approximations to an otherwise exact representation of the ground state of an anharmonic oscillator. The truncated wavefunctions are neither normalisable nor unique. Quite different such wavefunctions can give very accurate estimates for the energy eigenvalue, which are always finite. Possible parallels in quantum optics are drawn.
\end{abstract}

The coupled cluster method (CCM) [1-3] is widely recognised as providing one of the most broadly applicable and most accurate of all currently available formalisms to deal with the quantum manybody problem. It has been applied to a diverse range of condensed matter problems including finite nuclei [4], nuclear matter [5], the one-component fermion Coulomb plasma (or "electron gas") [6], and systems in quantum chemistry $[7,8]$. In each such case the results are at least as accurate as those obtained by any other method, except for the intrinsically exact Green function Monte Carlo or other stochastic simulation techniques that provide the benchmark results. The CCM is now also beginning to be applied with some considerable success to problems drawn from quantum field theory [9]. In view of this impressive corpus of successful applications it is interesting to study the fundamental properties of the method itself, and it is from this starting-point that the present study originated.

For present purposes we restrict the discussion only to the ground-state (g.s.) version of the so-called "normal" version of the CCM. As explained more fully below, this formalism is based on a very general "supercoherent" representation - the so-called $\exp (S)$ representation - of the exact ground state of

1 Permanent address: Departamento de Física Moderna, Facultad de Ciencias, Universidad de Granada, 18071 Granada, Spain. the quantum system, with respect to some appropriate model or reference state. Similar supercoherent states are also of particular topical interest in quantum optics $[10,11]$ as possible generalisations of the one-photon Glauber coherent states and twophoton or squeezed coherent states [12]. We return to this analogy at the end. The $\exp (S)$ representation was invented to incorporate fully the linked cluster theorem of Goldstone [3], and correspondingly to eliminate the unlinked diagrams of manybody perturbation theory that scale incorrectly with particle number, and whose appearance thereby leads to the so-called size-extensivity problem [13]. For applications to many-body or field-theoretical problems, this feature is crucial for practical implementation of the method.

Another feature of the CCM is that it is very strongly tied to the Schrödinger equation. In particular, the g.s. energy for example may thereby be calculated other than as the direct expectation value of the hamiltonian. Furthermore, and related to this, the requirement of normalisability of the exact ground state is never explicitly imposed. Normally in solving for the bound states of a quantum-mechanical hamiltonian, one knows that the requirement of normalisability of the states plays a key role in uniquely determining them and their eigenergies. The question may thus be raised as to the possibility, at least in principle, of the CCM leading to non-normalisable and non-unique states. It is the purpose of 
this Letter to address this latter question as of interest in its own right and extending beyond the confines of the CCM itself.

To this end we step back from many-body applications and study instead the very simple but important one-body model of the one-dimensional quartic anharmonic oscillator as a concrete example with which to elaborate on the above points. We first introduce the usual (oscillator or bosonic) creation and destruction operators $a^{\dagger}$ and $a$ respectively, which obey the usual canonical commutation relation $\left[a, a^{\dagger}\right]=1$, and the corresponding "bare" vacuum state $|0\rangle$, defined by $a|0\rangle=0$. The model hamiltonian is then given as,

$H=\frac{1}{2}+a^{\dagger} a+\frac{1}{4} \lambda\left(a+a^{\dagger}\right)^{4}, \quad \lambda>0$.

Its exact ground state $|\psi\rangle$ and corresponding g.s. energy $E$ are given by the Schrödinger equation

$H|\psi\rangle=E|\psi\rangle$.

A useful first step is to perform a Bogoliubov transformation to a new set of canonical operators $b$ and $b^{\dagger}$,

$b=\left(1-t^{2}\right)^{-1 / 2}\left(a-t a^{\dagger}\right), \quad|t|<1$.

While not essential for the basic CCM procedure described below, an optimal choice of the parameter $t$ in this change of basis can greatly accelerate the convergence of the resultant $\mathrm{CCM}$ equations relative to their counterparts built from the original operators $a$ and $a^{\dagger}$. This is particularly true for large values of the coupling constant $\lambda$. The new operators obey the same basic commutation relation $\left[b, b^{\dagger}\right]=1$ for arbitrary real parameter $t$ with $-1<t<1$, and have a new vacuum state $|\phi\rangle$ such that $b|\phi\rangle=0$. One can show that the corresponding relation between the two (normalised) vacua is,

$|\phi\rangle=\left(1-t^{2}\right)^{-1 / 4} \exp \left(\frac{1}{2} t a^{\dagger 2}\right)|0\rangle$.

Hence $|\phi\rangle$ is a squeezed vacuum state. It corresponds to a gaussian wavefunction in configuration space with a width distorted from that of the bare vacuum $|0\rangle$. The so-called Hartree or gaussian approximation is now obtained by choosing $t=\tilde{t}$ such that the variational estimate $E_{0}=E_{0}(t)=\langle\phi|H| \phi\rangle$ for the g.s. energy is minimised. With this choice of parameter $t$ one can further show that the hamiltonian of eq. (1) may be re-expressed in normal- ordered form in terms of the new operators as,

$H=E_{0}(\tilde{t})+\omega(\tilde{t}) b^{\dagger} b+\gamma(\tilde{t}):\left(b+b^{\dagger}\right)^{4}:$,

with easily derived expressions for the constants $E_{0}$, $\omega$ and $\gamma$ which we do not quote.

The g.s. CCM now exactly parametrises the ground state $|\psi\rangle$ of $H$ in the supercoherent form,

$|\psi\rangle=\exp (\hat{S})|\phi\rangle, \quad \hat{S}=\sum_{n=1}^{\infty} S_{n}\left(b^{\dagger}\right)^{n}$,

and the solution of the Schrödinger equation (2) is reduced to determining the (c-number) correlation coefficients $\left\{S_{n}\right\}$. We note that with the symmetric hamiltonian of eq. (1), the fact that $|\psi\rangle$ is a parity eigenstate allows us to impose the constraint that all coefficients $S_{n}$ with $n$ odd vanish. We also point out that we could choose, as in ref. [14], to carry out a more general calculation in which the amplitudes $S_{n}$ with $n$ odd are not set to zero at the start. In this case we would find for such spatially symmetric hamiltonians as that of eq. (1), that the resultant $\mathrm{CCM}$ equations discussed below trivially permit the solution with the odd amplitudes identically zero. This must obviously be true in the exact (untruncated) formalism, but it is also true at every level in the practical SUB $n$ truncation scheme discussed below. We should also note that, in principle, other (nonparity-conserving) solutions could exist, but for present purposes we restrict ourselves from the outset to parity eigenstates.

The g.s. energy $E$ and the non-vanishing (even-indexed) coefficients $S_{n}$ are formally determined by projecting eq. (2) onto the complete basis of states $\langle 0| b^{n} \exp (-\hat{S}), n=0,1,2, \ldots$,

$$
\begin{aligned}
& \left\langle\phi\left|\mathrm{e}^{-S} H \mathrm{e}^{s}\right| \phi\right\rangle=\left\langle\phi\left|H \mathrm{e}^{s}\right| \phi\right\rangle=E, \\
& \left\langle\phi\left|b^{n} \mathrm{e}^{-s} H \mathrm{e}^{s}\right| \phi\right\rangle=0, \quad n=2,4, \ldots .
\end{aligned}
$$

Use of the nested-commutator expansion for the factor $\mathrm{e}^{-\delta} \mathrm{He}^{s}$ and the explicit form of eq. (5) for $H$, leads easily to the expression for the g.s. energy,

$E=E_{0}(\tilde{t})+12 \gamma(\tilde{t})\left(S_{2}^{2}+2 S_{4}\right)$,

and, after a lengthy calculation, to the infinite system of coupled finite-order multinomial equations for the coefficients $\left\{S_{n}\right\}$, 


$$
\begin{aligned}
\delta_{n 4} & +(n+1)(n+2)(n+3)(n+4) S_{n+4} \\
& +4 n(n+1)(n+2) S_{n+2} \\
& +n[\omega(\tilde{t}) / \gamma(\tilde{t})+6(n-1)] S_{n}+4(n-2) S_{n-2} \\
& +\sum_{j, k} j k\{[2(j-1)(j-2)+3(j-1)(k-1) \\
& +2(k-1)(k-2)] \delta_{j+k, n+4}+6(j+k-2) \delta_{j+k, n+2} \\
& \left.+6 \delta_{j+k, n}\right\} S_{j} S_{k} \\
& +2 \sum_{j, k, l} j k l\left[(j+k+l-3) \delta_{j+k+l, n+4}\right. \\
& \left.+2 \delta_{j+k+l, n+2}\right] S_{j} S_{k} S_{l} \\
& +\sum_{j, k, l, m} j k l m \delta_{j+k+l+m, n+4} S_{j} S_{k} S_{l} S_{m} \\
& =0, \quad n=2,4, \ldots,
\end{aligned}
$$

where the summations over $j, k, l$ and $m$ run over the even positive integers.

The exact solution for the set of coefficients $\left\{S_{n}\right\}$ to the coupled system of equations (10) would thus produce both the exact (unique and normalisable) g.s. wavefunction of $H$ via eq. (6), and the exact g.s. energy $E$ via eq. (9). In practice however the infinite system of equations (10) must be approximated by truncation. A natural way to do this, referred to as the SUB $n$ approximation scheme, is simply to set all $S_{i}=0$ for $i>n$, and to solve the remaining equations numerically. It has been shown elsewhere [14,15] that this scheme is capable of giving extremely precise results for $E$ for even quite low values of the truncation index $(n \geqslant 6)$ for all values of the coupling constant $\lambda$. For $n=6$, for example, the accuracy is better than about $0.01 \%$ for all $\lambda$.

If the operator $S$ is not truncated, the wavefunction of eq. (6) is certainly an exact representation of any state not orthogonal to $|\phi\rangle$, and the untruncated equations (10) must have the exact $\left\{S_{n}\right\}$ as a solution for the exact $|\psi\rangle$. Whether this is the only solution is not clear, but it seems unlikely in view of the fact that the requirement that the norm of $|\psi\rangle$ be finite has not been imposed. At finite SUB $n$ truncations the situation is even more interesting since for all (finite) $n>2$ the SUB $n$-truncated wavefunction has infinite norm. Indeed even the SUB2-truncated wavefunction is normalisable only for $\left|S_{2}\right|<\frac{1}{2}$ (cf. eq. (4)).
It must be emphasised that this non-normalisability of the truncated $\mathrm{CCM}$ wavefunctions is of no concern when calculating the energy $E$ via eq. (9), since the method of derivation via eq. (7) has avoided taking an expectation value of $H$. We point out that there also exist techniques [16] for calculating the expectation values of arbitrary operators within the CCM which likewise can remain finite even when the norm is undefined. Thus, we do not regard this lack of normalisability of the SUB $n$-truncated wavefunctions as being at all serious in this context. In particular there exist large classes of bra vectors in the dual Hilbert space whose scalar products with the SUB $n$-truncated ket vector are completely well-defined and convergent. In practice the CCM is so arranged as to deal only with such finite expressions. Nevertheless, the SUB $n$-truncated system of equations (10) can, in principle, have a multiplicity of different solutions. We may regard such non-unique solutions as a manifestation of the nonnormalisability and the fact that we are thus working not in the usual Hilbert space but in some appropriately enlarged space.

We have described elsewhere [14] a numerical method of solution for the SUB $n$-truncated equations (10). For the hamiltonian of eq. (1) we defined a so-called "standard" solution with the following properties: (i) the g.s. energy $E$ is generated very accurately; (ii) the convergence of $E$ with increasing truncation index $n$ is initially very rapid, although the rate of convergence slows appreciably beyond some value of $n$ which depends on $\lambda$ [14]; (iii) the coefficients $S_{m}$ decrease rapidly in magnitude with increasing index $m$, at least for $m$ not too high; and (iv) small variations in the coupling parameter $\lambda$ and incremental changes in the truncation index $n$ produce smooth variations in the approximate solutions $\left\{S_{m}\right\}$. Our standard solution is also the thermodynamically stable solution as discussed by Kaulfuss and Altenbokum [15] in this context. We also presented in ref. [14] a single example of a second ("non-standard") solution in the specific case of SUB 18 approximation and for $\lambda=0.1$.

We have since explored several specific numerical procedures which generate many more examples of such alternative non-standard solutions, both for various values of the truncation index and over the whole range of coupling constants (as well as for an- 
harmonicities more general than quartic). The solutions thus generated are typically ill-mannered with respect both to small changes in the potential and to incremental changes in the truncation index. This behaviour is indicative of the co-existence of several branches of solutions, especially when coupled with the fact that these non-standard solutions are usually quite difficult to obtain numerically (both in terms of finding them at all and with respect to numerical convergence). Furthermore these alternative solutions for $\left\{S_{m}\right\}$ decrease much more slowly in magnitude with increasing index $m$, than is the case for the corresponding standard solution. However, these solutions very often have the striking feature that the corresponding g.s. energy $E$, calculated from eq. (9), is close to the exact result. Indeed sometimes the result is extremely accurate. Conversely, only rarely is it a poor approximation.

We compare in table 1 the non-standard solution obtained in ref. [14] for the case $\lambda=0.1$ in SUB18 approximation, with both the corresponding standard solution and a further new non-standard solution obtained by a different iterative procedure on the truncated CCM equations. While the three estimates for the g.s. energy $E$ are all quite close to the exact value of 0.55915 , the three solutions for the g.s. wavefunction are seen to be very different.

We show further results in table 2 for the new nonstandard solution for the case $\lambda=100$, and again

\section{Table 1}

Comparison of ground state correlation coefficients $\left\{S_{i}\right\}$ for SUB 18 , with $\lambda=0.1$, for the quartic anharmonic oscillator. Case 1 results are for the standard solution, case 2 results are for the non-standard solution of ref. [14] and case 3 results are for a new non-standard solution. The respective errors produced in $E$ are $-5.5 \times 10^{-7} \%, 0.03 \%$ and $-3.99 \%$.

\begin{tabular}{|c|c|c|c|}
\hline \multirow[t]{2}{*}{$i$} & \multicolumn{3}{|l|}{$S_{i}$} \\
\hline & case 1 & case 2 & case 3 \\
\hline 2 & $1.60 \times 10^{-3}$ & $-1.75 \times 10^{-3}$ & $-4.02 \times 10^{-2}$ \\
\hline 4 & $-2.89 \times 10^{-3}$ & $-2.45 \times 10^{-3}$ & $-5.92 \times 10^{-2}$ \\
\hline 6 & $7.41 \times 10^{-5}$ & $1.42 \times 10^{-4}$ & $3.10 \times 10^{-2}$ \\
\hline 8 & $-2.65 \times 10^{-6}$ & $-4.64 \times 10^{-4}$ & $2.29 \times 10^{-3}$ \\
\hline 10 & $1.09 \times 10^{-7}$ & $-3.96 \times 10^{-5}$ & $-2.66 \times 10^{-3}$ \\
\hline 12 & $-4.90 \times 10^{-9}$ & $4.12 \times 10^{-5}$ & $3.54 \times 10^{-4}$ \\
\hline 14 & $2.32 \times 10^{-10}$ & $-6.12 \times 10^{-6}$ & $-1.99 \times 10^{-4}$ \\
\hline 16 & $-1.08 \times 10^{-11}$ & $-2.15 \times 10^{-7}$ & $8.84 \times 10^{-5}$ \\
\hline 18 & $3.58 \times 10^{-13}$ & $1.19 \times 10^{-7}$ & $-8.08 \times 10^{-6}$ \\
\hline
\end{tabular}

compare with the standard solution. The sensitivity of the results on the SUBn truncation is also displayed for both solutions. We note from table 2 that as the truncation index $n$ is increased, at least the lowest few correlation coefficients $S_{m}$ for the nonstandard solution seem to be approaching those of the standard solution. On the other hand, the actual convergence properties of the coefficients $\left\{S_{m}\right\}$ for even the standard solution, as the SUB $n$ truncation index $n$ is increased without bound, are still far from clear. Although of interest in its own right, this problem is not of direct concern to us here however.

In conclusion, it seems that the non-normalisability of the SUB $n$-truncated supercoherent wavefunctions results in the non-uniqueness of the approximate representation for the wavefunction. The number of possible such solutions grows without bound as $n$ is increased. Furthermore, at least some of this class of solutions for a given potential and at a fixed level of approximation, give very similar results for the corresponding eigenvalue $E$. We may conjecture that as $n \rightarrow \infty$ we arrive at an infinite set of different solutions for $|\psi\rangle$, only one of which is normalisable, but all of which give the same (exact) energy $E$ when calculated via the CCM procedure of eq. (7).

Finally we remark that the use of such supercoherent states may also find applications in quantum optics. For example the SUB1 and SUB2 states are respectively just the usual Glauber coherent states and, for $\left|S_{2}\right|<\frac{1}{2}$ the squeezed coherent states [12]. For these two cases our supercoherent states may also be represented as an appropriate unitary (displacement or squeezing) operator acting on the vacuum $|\phi\rangle$. Such a unitary mapping of the state $|\phi\rangle$ of course preserves its norm. Conversely, in SUBn truncations with $n>2$ (or with $\left|S_{2}\right|>\frac{1}{2}$ for $n=2$ ) our supercoherent states are not normalisable and such unitary operators cannot be defined. It is only within such a unitary framework that D'Ariano et al. [11] have proved the impossibility of naively generalising the squeezed $(n=2)$ two-photon coherent states to normalisable $(n>2)$ multi-photon coherent states. We have seen that while the CCM is not a manifestly norm-preserving (unitary) transformation from the model wavefunction $|\phi\rangle$, physical quantities are nevertheless well-defined and finite. We hope to return in a later publication to possible applications of 
Table 2

Comparison of standard (S) and non-standard (N) solutions for the quartic anharmonic oscillator, with $\lambda=100$, in various SUB $n$ approximations. The last line shows the respective percentage errors in the energy $E$.

\begin{tabular}{|c|c|c|c|c|c|c|}
\hline \multirow[t]{3}{*}{$i$} & \multicolumn{6}{|l|}{$S_{i}$} \\
\hline & \multicolumn{2}{|l|}{ SUB18 } & \multicolumn{2}{|l|}{ SUB20 } & \multicolumn{2}{|l|}{ SUB22 } \\
\hline & $\mathbf{N}$ & $S$ & $\mathbf{N}$ & $\mathbf{S}$ & $\mathbf{N}$ & $\mathbf{S}$ \\
\hline 2 & $9.56 \times 10^{-3}$ & $6.99 \times 10^{-3}$ & $9.60 \times 10^{-3}$ & $8.91 \times 10^{-3}$ & $9.66 \times 10^{-3}$ & $9.53 \times 10^{-3}$ \\
\hline 4 & $-7.33 \times 10^{-3}$ & $-5.86 \times 10^{-3}$ & $-7.34 \times 10^{-3}$ & $-6.61 \times 10^{-3}$ & $-7.37 \times 10^{-3}$ & $-7.03 \times 10^{-3}$ \\
\hline 6 & $3.97 \times 10^{-4}$ & $4.17 \times 10^{-4}$ & $3.93 \times 10^{-4}$ & $3.05 \times 10^{-4}$ & $3.90 \times 10^{-4}$ & $3.19 \times 10^{-4}$ \\
\hline 8 & $-2.90 \times 10^{-5}$ & $-1.67 \times 10^{-4}$ & $-2.72 \times 10^{-5}$ & $-6.92 \times 10^{-5}$ & $-2.40 \times 10^{-5}$ & $-3.49 \times 10^{-5}$ \\
\hline 10 & $2.64 \times 10^{-6}$ & $3.54 \times 10^{-5}$ & $2.40 \times 10^{-6}$ & $1.76 \times 10^{-5}$ & $1.76 \times 10^{-6}$ & $8.31 \times 10^{-6}$ \\
\hline 12 & $-2.64 \times 10^{-7}$ & $-2.71 \times 10^{-6}$ & $-2.74 \times 10^{-7}$ & $-2.20 \times 10^{-6}$ & $-2.38 \times 10^{-7}$ & $-1.33 \times 10^{-6}$ \\
\hline 14 & $2.31 \times 10^{-8}$ & $-2.61 \times 10^{-7}$ & $3.09 \times 10^{-8}$ & $5.48 \times 10^{-8}$ & $3.88 \times 10^{-8}$ & $1.04 \times 10^{-7}$ \\
\hline 16 & $-1.47 \times 10^{-9}$ & $8.12 \times 10^{-8}$ & $-2.73 \times 10^{-9}$ & $2.54 \times 10^{-8}$ & $-4.83 \times 10^{-9}$ & $2.12 \times 10^{-9}$ \\
\hline 18 & $5.27 \times 10^{-11}$ & $-6.97 \times 10^{-9}$ & $1.67 \times 10^{-10}$ & $-4.34 \times 10^{-9}$ & $4.24 \times 10^{-10}$ & $-1.55 \times 10^{-9}$ \\
\hline 20 & & & $-5.76 \times 10^{-12}$ & $2.95 \times 10^{-10}$ & $-2.55 \times 10^{-11}$ & $1.90 \times 10^{-10}$ \\
\hline 22 & & & & & $8.82 \times 10^{-13}$ & $-1.08 \times 10^{-11}$ \\
\hline$\%$ & $4.94 \times 10^{-3}$ & 0.391 & $2.92 \times 10^{-3}$ & 0.197 & $-4.16 \times 10^{-3}$ & 0.085 \\
\hline
\end{tabular}

the present approach to define multi-photon coherent states.

We are pleased to acknowledge the support for this work of the British/Spanish Joint Research Programme (Acciones Integradas), administered jointly by the British Council and the Spanish Ministry of Education and Science. R.F.B. acknowledges the support of a research grant from the U.K. Science and Engineering Research Council, and M.C.B. acknowledges the support of CICYT (contract 1234/ 84) and the Dirección General de Cooperación Internacional.

\section{References}

[1] F. Coester, Nucl. Phys. 7 (1958) 421.

[2] R.F. Bishop and K.H. Lührmann, Phys. Rev. B 17 (1978) 3757.
[3] R.F. Bishop and H. Kümmel, Phys. Today 40 (1987) 52.

[4] H. Kümmel, K.H. Lührmann and J.G. Zabolitzky, Phys. Rep. C 36 (1978) 1.

[5] B.D. Day and J.G. Zabolitzky, Nucl. Phys. A 366 (1981) 221.

[6] R.F. Bishop and K.H. Lührmann, Phys. Rev. B 26 (1982) 5523.

[7] V. Kvasnicka, V. Laurinc and S. Biskupic, Phys. Rep. 90 (1982) 160.

[8] K. Szalewicz, J.G. Zabolitzky, B. Jeziorski and H.J. Monkhorst, J. Chem. Phys. 81 (1984) 2723.

[9] M. Funke, U. Kaulfuss and H. Kümmel, Phys. Rev. D 35 (1987) 621.

[10] R.A. Fisher, M.M. Nieto and V.D. Sandberg, Phys. Rev. D 29 (1984) 1107.

[11] G. D'Ariano, M. Rasetti and M. Vadacchino, Phys. Rev. D 32 (1985) 1034.

[12] H.P. Yuen, Phys. Rev. A 13 (1976) 2226.

[13] R.J. Bartlett and G.D. Purvis, Phys. Scr. 21 (1980) 255.

[14] R.F. Bishop and M.F. Flynn, Phys. Rev. A (1988), to be published.

[15] U.B. Kaulfuss and M. Altenbokum, Phys. Rev. D 33 (1986) 3658.

[16] J. Arponen, Ann. Phys. (NY) 151 (1983) 311. 